\title{
Strontium ranelate: ready for clinical use as disease-modifying osteoarthritis drug?
}

\author{
Floris P J G Lafeber, ${ }^{1}$ Jacob M van Laar $^{2}$
}

In the past decade, clinical practice in rheumatology has been transformed by the advent of 'biologicals'. The use of these new drugs has significantly improved the outcome of patients with rheumatoid arthritis especially for those refractory to (patient-tailored) conventional diseasemodifying antirheumatic drug (DMARD) therapy. The question is, is it now time for a gap-bridging, large step forward in the treatment of an even more commonly seen rheumatic disease, osteoarthritis? Has the era of the disease-modifying osteoarthritis drug (DMOAD) begun? Is 'OA the new RA' (quote from Professor P Conaghan, 2012 annual meeting of the British Society of Rheumatology (BSR)), or is this still wishful thinking?

It no longer needs proclamation of worldwide numbers of patients who suffer from non-attendance and decreased productivity at work and unemployment, or costs of medical and paramedical care, medication use, and surgical treatments with joint replacement in the end, to recognise that osteoarthritis is a huge socioeconomic problem. ${ }^{1}$ Osteoarthritis is the most common disabling joint disease worldwide. Moreover, its incidence and prevalence are increasing because of ageing, higher life expectancy, and lifestyle changes, leading to a growing population of patients with osteoarthritis. ${ }^{2}$

Osteoarthritis is clinically characterised primarily by joint pain and consequently functional limitations. Cartilage degradation is still considered pivotal in its pathology. This might be considered surprising, since cartilage is not innervated, so osteoarthritic pain must arise from other tissues involved. Changes in periarticular bone, synovial tissue and other periarticular soft

${ }^{1}$ Rheumatology \& Clinical Immunology, University Medical Centre Utrecht, Utrecht, The Netherlands ${ }^{2}$ Musculoskeletal Research Group, Institute of Cellular Medicine, Newcastle University, Newcastle upon Tyne, UK

Correspondence to Professor Floris P J G Lafeber, UMC Utrecht, Rheumatology \& Clinical Immunology, Utrecht 3508 GA, The Netherlands;

f.lafeber@umcutrecht.nl

FPJGL and JMvL contributed equally. tissue structures such as ligaments and muscles are inextricably related to the osteoarthritic process. Obviously, all these structural changes are part of a cascade that eventually leads to overt osteoarthritis, and they need to be considered in unison when related to clinical symptoms. The sequence and relative importance of each of these processes may differ between individuals, between joints, between disease stages, and over time. ${ }^{2}$ Appreciating this, it might well be that not all patients at all phases in their disease need the same treatment approach.

The growing body of literature on osteoarthritis shows that this disease is still a major challenge for scientists as much as clinicians. Early in the disease, general practitioners and rheumatologists may be able to help with nonpharmacological therapy combined with pain medication and, when necessary, anti-inflammatory drugs, but these will not stop the degenerative process. ${ }^{3}$ In end-stage disease, orthopaedic interventions are effective for a certain time period but are not curative; joint replacement is the final solution. Within these boundaries of early and late stage treatment, there is a huge gap where there is an urgent need for new therapies, particularly treatment modalities with structure-modifying properties ${ }^{4}$ (figure 1 ). It will be a challenge for rheumatologists and orthopaedic surgeons in the coming decade to jointly fill this gap.

Early attempts to modify tissue structure in osteoarthritis were hampered by a lack of proper biochemical and imaging markers to evaluate functional joint tissue repair. In response, more effort was put into improving surrogate markers to demonstrate more subtle changes in tissue repair. This field is making progress, ${ }^{5} 6$ but the steps made are small, and implementation in trials and clinical practice is costly. Large-scale, lengthy, and therefore costly, clinical trials are still required to demonstrate tissue structure modification. Nevertheless, the need to find a solution to the huge socioeconomic problem of joint degeneration has encouraged some to persevere in their search for joint tissue repair modalities. However, at present, the regulatory authorities have still not approved any of the current potential DMOAD treatments, as researchers have failed to provide evidence of both structural and clinical improvement as laid out in guidelines. ${ }^{7}$ But, maybe, the quest for DMOADs has now become fruitful.

Annals of Rheumatic Diseases features potentially the first DMOAD. The first data of a large double-blind, randomised placebo-controlled clinical trial on the 'Efficacy and safety of strontium ranelate in the treatment of knee osteoarthritis' (SEKOIA; controlled Strontium ranelate Efficacy in Knee Osteoarthritis triAl), sponsored by Servier (Suresnes, France), are presented by Reginster et al. ${ }^{8}$

Strontium ranelate is licensed for the treatment of osteoporosis. The most commonly used osteoporosis treatments (oestrogen replacement therapy, selective oestrogen-receptor modulators, bisphosphonates and calcitonin) act by inhibiting bone resorption. Strontium ranelate, in contrast, may have a dual mode of action, simultaneously increasing bone formation while decreasing bone resorption. ${ }^{9}$

There was a rationale for testing this anti-osteoporotic drug for the treatment of osteoarthritis. ${ }^{10}$ In post hoc analyses of two large osteoporosis studies, strontium ranelate was suggested to beneficially influence cartilage in the osteoarthritic population within these osteoporosis cohorts. Strontium ranelate-treated patients with a history of clinical osteoarthritis (vertebral, hip, knee, hand or other) had a significant decrease in urinary collagen type II C-terminal telopeptide (CTX-II), considered a marker of cartilage degradation, compared with placebo-treated patients. ${ }^{11}$ However, surprisingly (to us at least), the nonosteoarthritic patients who were not expected to have cartilage degeneration, also showed a clear decrease in CTX-II levels. The reason for this may be that CTX-II is now no longer considered a marker of cartilage damage alone, but may also be released from bone. ${ }^{12}$ In trials of the anti-osteoporotic drug, risedronate, in patients with knee osteoarthritis, urinary CTX-II levels also decreased significantly over time, but no beneficial effect of risedronate on joint space narrowing (JSN) was observed. ${ }^{13}$ This raises questions as to whether a decrease in urinary CTX-II levels can be used as a reliable marker for chondroprotection per se.

In another post hoc evaluation of cohorts of patients with high risk of, or 


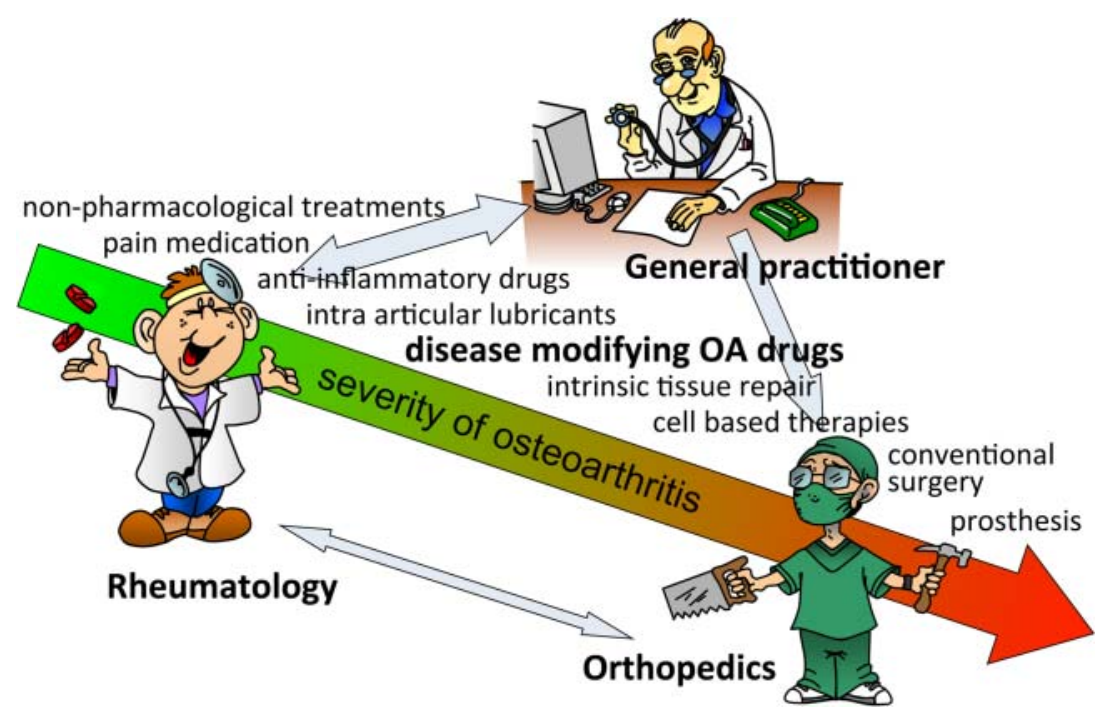

Figure 1 The gap in treatment modalities between rheumatology and orthopaedics. $O A$, osteoarthritis.

prevalent, vertebral fractures, radiographs were taken for detection of these fractures. These radiographs were reused to identify patients with radiographic lumbar spine osteoarthritis, based on a combined score of JSN and osteophytes. ${ }^{14}$ After 3 years of treatment with strontium ranelate, $10 \%$ experienced an increase in the overall osteoarthritis score versus $17 \%$ in the placebo-treated group. For JSN alone, this was $8 \%$ and $12 \%$, respectively. This more convincingly argues for a chondroprotective role of strontium ranelate than a change in CTX-II levels. However, it should be kept in mind that radiographs not taken in a highly standardised way for the purpose of evaluating radiographic characteristics of osteoarthritis should be interpreted with caution. ${ }^{15}$

The fact that osteoarthritis is increasingly considered a disease in which periarticular bone changes take a central role was an additional motivation to test strontium ranelate for treatment of this disease. For example, oedema-like bone marrow lesions (BMLs) are considered to be associated with articular cartilage loss $^{16}$ and, most importantly, with pain sensation. ${ }^{17}$

In vitro experiments suggested direct effects of strontium ranelate on cartilage as well. Strontium ranelate increased cartilage matrix formation by isolated chondrocytes by stimulating anabolism without influencing the (interleukin $1 \beta$ induced) catabolic pathways. ${ }^{18}$ In this way, it could restore the imbalance between cartilage matrix formation and breakdown as observed during osteoarthritis. This positive effect on anabolism fits with the observation that strontium ranelate increases serum insulin-like growth factor 1 (IGF-1) levels in osteoporotic postmenopausal women, ${ }^{19}$ IGF-1 being a known stimulant of cartilage matrix synthesis. ${ }^{20}$ Of note, it had already been observed that strontium might directly influence cartilage back in 1985. ${ }^{21}$ In strontium-induced rickets in rats (characterised by bone 'softening'), an increased aggregability of proteoglycan monomers with hyaluronate was found in the epiphyseal growth plate. It was suggested that strontium not only has an effect on bone but also induces chondrocytes to produce a matrix with a different macromolecular composition.

Apparently, this was sufficient for the SEKOIA investigators and the trial's sponsor to start a large-scale, prospective, multicentre, international, double-blind, placebo-controlled trial of strontium ranelate in patients with knee osteoarthritis. ${ }^{10}$ Despite some changes in design during the study, ${ }^{8}$ this study can be classified as well designed, with appropriate data collection and analyses. In the context of discussions on the, in general, better outcomes of industry-driven versus investigator-driven DMOAD studies, the role of the sponsor is clearly described. ${ }^{8}$

After slightly less than 5 years, the last of more than 1000 patients completed the 3-year follow-up. Loss to follow-up was high, but in the same range as in other large trials in osteoarthritis. ${ }^{22}$ Unfortunately, the reasons for loss to follow-up were not provided in detail. Most importantly, good care was taken to obtain optimal standardisation during acquisition of radiographs to prevent disappointment previously observed in such large-scale DMOAD studies. ${ }^{23}$ As such, the outcome provides convincing radiographic data demonstrating that strontium ranelate treatment for 3 years results in less progression of radiographic JSN (the primary outcome of the study). Also, when analysed by numbers of patients with more than $0.5 \mathrm{~mm}$ progression in JSN, differences were (statistically) significantly in favour of the strontium ranelate groups. Although the authors state that there was no statistically significant difference between the two doses, the lower dose of $1 \mathrm{~g} /$ day was more effective in slowing down JSN than the higher dose of $2 \mathrm{~g} /$ day.

In addition to this structure-modifying activity, the drug had a beneficial effect on symptoms, albeit modest. There was hardly any effect on the overall Western Ontario and McMaster Universities Arthritis Index (WOMAC) score or WOMAC pain score, but after 3 years the symptomatic benefit of the higher $2 \mathrm{~g} /$ day dose was significantly different from placebo. The study showed a marked placebo effect over the whole 3-year study period, and, although in line with results from earlier osteoarthritis trials, ${ }^{24}$ this might have been a disadvantage in detecting clinical benefit of the drug.

The limited effect on pain relief is reflected by a lack of a difference between the treatment arms in concomitant medication use during the study period. It is tempting to speculate that the cartilage structure-modifying effect of this drug might have, at least in part, benefitted from the limited effect on pain relief. The recent tanezumab (anti-nerve growth factor) studies suggest that significant pain relief may induce progressive cartilage tissue structure damage. ${ }^{25}$ It could well be that significant pain relief alone encourages more intensive use of a compromised joint, leading to further wear and tear of the already damaged cartilage and, with that, progression of radiographic JSN. On the other hand, pain relief may improve training and, with that, the strength of ligaments and muscles, increasing joint stability, protecting against wear and tear of cartilage and, with that, JSN. This balance between favourable joint use and harmful overloading is fundamental in treatment of osteoarthritis. Clearly, the complex relationship between pain and cartilage degeneration poses challenges for any DMOAD clinical development programme. There may be rather different aetiological pathways in structural and symptomatic osteoarthritis. This raises 
the question as to whether both cartilage structure modification and pain relief can be gained by a single drug or whether a combination of drugs is necessary to tackle both, in the light of requirements by the regulatory authorities for cartilage tissue structure modification to be necessarily accompanied by pain relief. On the other hand, cartilage damage will contribute to clinical symptoms. In that case, cartilage tissue structure repair should, at least over time (but probably not directly), lead to a reduction in pain.

A key question is, will patients be persuaded to take strontium ranelate-a drug with a risk, albeit low, of allergy and thromboembolism ${ }^{26}$-for prolonged periods of time, knowing that the effects on cartilage damage take time and may not have immediate clinical benefit? The authors state that the observed $27 \%$ reduction in radiological JSN translates into saving nearly a year of average radiological osteoarthritis progression over 3 years. This implies that, on average, 14 patients would need to be treated over 3 years to prevent a single case of radiological progression of more than $0.5 \mathrm{~mm}$, a threshold predicting osteoarthritisrelated surgery. It is of interest to study post hoc or upon extended follow-up whether this benefit actually prevents or delays joint replacement surgery. But, with minimal clinical benefit over 3 years use, would patients be compliant in taking daily medication with these drug characteristics? Moreover, which dose is recommended to treat patients? A low dose of strontium ranelate induces a DMOAD effect, but the symptomatic effect is present with the high dose. Perhaps it is possible to start administration of the drug at high dose to control pain and then to reduce the dose to control progression of cartilage loss. On the other hand, considering the side effects, the low dose to slow down joint damage might be combined with other strategies to diminish pain. As mentioned above, we might be expecting too much from a single drug to encompass both.

Answers to these questions require further studies which build on the results of the SEKOIA trial. The identification of strontium ranelate as a DMOAD is a milestone in osteoarthritis research, but we still have some miles to go, and future (post hoc and new) studies on this drug are needed to consolidate this step forward in the treatment of osteoarthritis. Pressing questions are, for which patients (which phenotype ${ }^{2}$ ) and at what stage of the disease is strontium ranelate most effective? Clearly, not all patients with osteoarthritis benefit from each specific treatment, so identification of predictive factors of (non)responsiveness would be of great help.

Being a modulator of bone turnover, strontium ranelate would be expected to act on the aberrant periarticular bone turnover in osteoarthritis. Although speculative, the drug may, via 'normalisation' of bone turnover, indirectly, mechanically and biochemically influence cartilage turnover. Diminished bone sclerosis may soften the bone matrix, providing better biomechanical conditions for the affected overlaying cartilage. Also, altered release of soluble mediators from the bone during treatment may influence cartilage beneficially. Using bone scintigraphy, Paul Dieppe demonstrated years ago that the activity of subchondral bone determines loss of cartilage. ${ }^{27}$ A clear role for involvement of periarticular bone would fit the observations from the recent zolendronate study. ${ }^{28}$ This study included 60 patients with evident BMLs assessed by MRI divided over two treatment arms with just 1-year follow-up, and demonstrated significant reduction in BML size accompanied by a decrease in pain.

On the other hand, direct effects on cartilage cannot be ruled out. In this context, it would have been informative if the authors had provided more data on bone changes; its importance could have been anticipated. The CTX-II data are suggestive, at least. Post hoc analyses may give us better insight into the characteristics of responders and nonresponders. In this respect, it might be relevant to take the osteoporotic status of the patients into account considering the supposedly mutual exclusion of the two disorders $^{29}$ and the mode of action of this specific drug. These analyses may be followed by smaller more-targeted studies on subpopulations, such as the zolendronate study. Only then can a full assessment of the drug's potential benefit in osteoarthritis be made. An additional advantage of such smaller studies is that more sophisticated analyses of joint tissue degeneration, such as MRI, can be incorporated, which is too costly in large cohort studies.

With respect to the underlying mechanisms of the structure-modifying properties of strontium ranelate, another paper in this issue of Annals of Rheumatic Diseases provides some clarification. Pelletier et $a l^{30}$ provide data on strontium ranelate in an animal model of osteoarthritis. They postulate that 'Strontium ranelate reduces the progression of experimental dog osteoarthritis by inhibiting the expression of key proteases in cartilage and of interleukin-1 $\beta$ in the synovium'.

The anterior cruciate ligament transection model was used in which permanent joint instability results in cartilage damage, evident changes in subchondral bone, and (depending on surgery) significant synovial inflammation. Importantly, owing to unloading of the affected unstable joint in this model, the osteopenic trabecular bone changes are extended to the metaphyseal area. ${ }^{31}$ This makes it not likely that the observed histological and biochemical changes in cartilage are secondary to the bone changes in this prevention protocol. Similarly, previous results on other osteoarthritis drugs by this group may have indirectly been caused by control of synovial inflammation rather than by direct effects on cartilage. The treatment, started shortly after surgery, might have prevented significant bone changes from occurring and, with that, diminished cartilage changes. This fits with the observed decrease in disorganisation of the cartilage collagen network in the strontium ranelate-treated animals. With the knowledge of the half life of collagen, this is clearly prevention, and not treatment, of the disease. A causal relation between changes in subchondral plate thickness and cartilage damage in animal models has been reported. ${ }^{32}$ As such, the diminished cartilage damage, as observed in this canine study, may be the result of prevention of subchondral bone plate thickening induced by strontium ranelate treatment of the animals. The absence of an effect on chondrocyte catabolism in previous studies with isolated chondrocytes $^{19}$ supports the idea that the observed decrease in cartilage catabolism by strontium ranelate in the present canine study is probably an indirect effect. Of interest in this respect is the recent observation that strontium ranelate stimulates differentiation of bone marrow mesenchymal stem cells into osteoblasts. ${ }^{33}$ Unfortunately, not all data on bone changes were provided in detail in this in vivo animal study, and more sophisticated analyses of bone by microCT ${ }^{31} 32$ or MRI - with which the group has extensive experience ${ }^{34}$ - were lacking. As for the clinical trial, no relations between bone and cartilage changes were provided.

A completely different, hypothetical and potentially additional effect of strontium ranelate may be related to its physicochemical properties. During treatment, some strontium is incorporated 
into hydroxyapatite, where it can substitute for up to 1 in 10 calcium atoms. This could in part explain the increase in bone mineral density in osteoporosis studies because strontium has a higher atomic number than calcium, with greater attenuation of the x-rays used for dual-energy $x$-ray absorptiometry (DEXA) measurements. ${ }^{35}$ Whether such a physicochemical activity of strontiumthat is, incorporation into cartilage matrix components-plays a role in the chondroprotective effects requires further study.

Two other DMOAD studies are presented in this issue. A well-designed and performed randomised, placebo-controlled, double-blind trial on the oral selective inhibitor of inducible nitric oxide synthase (iNOS), cindunistat (SD-6010), from Pfizer in almost 1500 patients did not demonstrate superiority of this drug over placebo on JSN in patients with evident (Kellgren and Lawrence (K\&L) grade 2-3) radiographic knee osteoarthritis. ${ }^{22}$ A retrospective evaluation of the use of statins in more than 2000 patients of the Osteoarthritis Initiative with suspected or confirmed knee osteoarthritis evaluated over 4 years revealed $20 \%$ statin users. ${ }^{36}$ However, statin use was not associated with improvement in knee pain, function or structural progression. This contrasts with a recent study in the Rotterdam cohort (where retrospective evaluation was carried out in almost 3000 patients): statin use (10\% of patients) was associated with reduced incidence and progression of knee osteoarthritis. ${ }^{37}$ This apparent discrepancy is extensively discussed and thought to be related to differences in the populations evaluated. Interestingly, in the iNOS inhibitor study, in a subgroup of patients, omitting those with the most severe damage (K\&L grade 3), after 1 year of treatment with $50 \mathrm{mg} /$ day cindunistat, JSN was statistically significantly ( $50 \%)$ less than in the placebo-treated patients. Clearly, the results of the studies on these two potential DMOADs also warrant more-targeted approaches in smaller more defined cohorts based on selection of specific distinct phenotypes and severity of disease from the general osteoarthritis patient population.

Clearly, none of the three drugs presented is ready for use as a DMOAD in clinical practice. The quest for an appropriate treatment of osteoarthritis between analgesics and pain medication on the one hand and surgical solutions on the other has only just begun. Modification of cartilage tissue structure might be obtained not only by DMOADs that interfere with (periarticular) bone turnover, but also by anti-inflammatory medication, via other mechanisms. ${ }^{4}$ Most recently, (apparently) effective antiinflammatory treatment of knee osteoarthritis by glucocorticoids has been reported, ${ }^{38}$ and more will definitely come. This supports the idea of differentiation of subpopulations of patients, providing more-targeted treatment during the variable process of this highly prevalent disease.

\section{Funding None}

\section{Competing interests None.}

\section{Provenance and peer review Commissioned;} externally peer reviewed.

Received 5 October 2012

Revised 8 November 2012

Accepted 15 November 2012



- http://dx.doi.org/10.1136/annrheumdis-2012-201710

- http://dx.doi.org/10.1136/annrheumdis-2012-202231

- http://dx.doi.org/10.1136/annrheumdis-2012-202239

Ann Rheum Dis 2013;72:157-161.

doi:10.1136/annrheumdis-2012-202453

\section{REFERENCES}

1. Hermans J, Koopmanschap MA

Bierma-Zeinstra SM, et al. Productivity costs and medical costs among working patients with knee osteoarthritis. Arthritis Care Res 2012;64:853-61.

2. Bijlsma JW, Berenbaum F, Lafeber FP. Osteoarthritis: an update with relevance for clinical practice. Lancet 2011;377:2115-26.

3. Jordan KM, Arden NK, Doherty M, et al. EULAR Recommendations 2003: an evidence based approach to the management of knee osteoarthritis: Report of a Task Force of the Standing Committee for International Clinical Studies Including Therapeutic Trials (ESCISIT). Ann Rheum Dis 2003;62:1145-55.

4. Mastbergen SC, Saris DBF, Lafeber FPJG. Functional tissue repair in our joints; is it here, near or never happening. Nature Rev Rheum 2012; In press.

5. Guermazi A, Roemer FW, Hayashi D. Imaging of osteoarthritis: update from a radiological perspective. Curr Opin Rheumatol 2011;23:484-91.

6. van Spil WE, DeGroot J, Lems WF, et al. Serum and urinary biochemical markers for knee and hip-osteoarthritis: a systematic review applying the consensus BIPED criteria. Osteoarthritis Cartilage 2010; 18:605-12.

7. FDA-Clinical development programs for drugs devices and biological products intended for the treatment of $\mathbf{O A}$. http://www.fda.gov/ downloads/Drugs/GuidanceComplianceRegulatory Information/Guidances (7/99).

8. Reginster J-Y, Badurski J, Bellamy N, et al. Efficacy and safety of strontium ranelate in the treatment of knee osteoarthritis: results of a double-blind, randomised placebo-controlled trial. Ann Rheum Dis 2013;72:179-86.

9. Marie PJ. Strontium ranelate: new insights into its dual mode of action. Bone 2007;40:S5-8.

10. Cooper C, Reginster J-Y, Chapurlat R, et al. Efficacy and safety of oral strontium ranelate for the treatment of knee osteoarthritis: rationale and design of randomised, double-blind, placebo-controlled trial. Curr Med Res Opin 2012;28:231-9.

11. Alexandersen P, Karsdal MA, Byrjalsen I, et al. Strontium ranelate effect in postmenopausal women with different clinical levels of osteoarthritis. Climacteric 2011;14:236-43.

12. Van Spill EW, Drossaers-Bakker KW, Lafeber FPJG. Associations of CTX-II with biochemical markers of bone turnover raise questions on its tissue origin: data from CHECK, a cohort study of early osteoarthritis. Ann Rheum Dis 2012; Jun 11. (Epub ahead of print)

13. Bingham CO, Buckland-Wright JC, Garnero P, et al. Risedronate decreases biochemical markers of cartilage degradation but does not decrease symptoms or slow radiographic progression in patients with medial compartment osteoarthritis of the knee: results of the 2-year multinational knee osteoarthritis structural arthritis study. Arthritis Rheum 2006;54:3494-507.

14. Bruyere 0, Delferriere D, Roux C, et al. Effects of strontium ranelate on spinal osteoarthritis progression. Ann Rheum Dis 2008;67:335-9.

15. Kinds MB, Vincken KL, Hoppinga TN, et al. Influence of variation in semiflexed knee positioning during image acquisition on separate quantitative radiographic parameters of osteoarthritis, measured by Knee Images Digital Analysis. Osteoarthritis Cartilage 2012;20:997-1003

16. Karsdal MA, Leeming DJ, Dam EB, et al. Should subchondral bone turnover be targeted when treating osteoarthritis? Osteoarthritis Cartilage 2008;16:638-46.

17. Roemer FW, Guermazi A, Javaid MK, et al. Change in MRI-detected subchondral bone marrow lesions is associated with cartilage loss: the MOST Study. A longitudinal multicentre study of knee osteoarthritis. Ann Rheum Dis 2009:68:1461-5.

18. Zhang $\mathbf{Y}$, Nevitt M, Niu J, et al. Fluctuation of knee pain and changes in bone marrow lesions, effusions, and synovitis on magnetic resonance imaging. Arthritis Rheum 2011;63:691-9.

19. Henrotin J-Y, LaBasse A, Zheng SX, et al Strontium ranelate increases cartilage matrix formation. $J$ Bone Miner Res 2001:16:299-308.

20. Gulhan I, Bilgili S, Gunaydin R, et al. The effect of strontium ranelate on serum insulin like growth factor- 1 and leptin levels in osteoporotic post-menopausal women: a prospective study. Arch Gynecol Obstet 2008;278:437-41.

21. Reinholt F, Engfeldt $B$, Heinegard $D$, et al. Proteoglycans and glycosaminoglycans of normal and strontium rachitic epiphyseal cartilage. Collagen Rel Res 1985:5:41-53.

22. Hellio Le graverand M-P, Clemmer RS, Redifer $P$, et al. A 2-year randomised, double-blind, placebo-controlled, multicentre study of oral selective iNOS inhibitor, cindunistat (SD-6010), in patients with symptomatic osteoarthritis of the knee. Ann Rheum Dis 2013;72:187-95.

23. Reginster JY, Deroisy R, Rovati LC, et al. Long-term effects of glucosamine sulphate on osteoarthritis progression: a randomised, placebo-controlled clinical trial. Lancet 2001;357:251-6; (and comments).

24. Zhang W, Robertson J, Jones AC, et al. The placebo effect and its determinants in osteoarthritis: meta-analysis of randomised controlled trials. Ann Rheum Dis 2008:67:1716-23.

25. Ackermann PW. Letter to the editor regarding Katz et al., Efficacy and safety of tanezumab in the treatment of chronic low back pain (Pain 2011;152:2248-2258) and Hill, Blocking the effects of NGF as a route to safe and effective pain relieffact or fancy? (Pain 2011;152:2200-2201). Pain 2012;153:1128-31.

26. EMA. March 15 2012: Questions and answers on the review of Protelos and Osseor (strontium 
ranelate). Outcome of procedures under Article 20 of Regulation (EC) No 726/2004. http://www.ema europa.eu/docs/...../Medicine OA/2012/03/ WC500124208.pdf

27. Dieppe $\mathbf{P}$, Cushnaghan J, Young P, et al. Prediction of the progression of joint space narrowing in osteoarthritis of the knee by bone scintigraphy. Ann Rheum Dis 1993;52:557-63.

28. Laslett LL, Doré DA, Quinn SJ, et al. Zoledronic acid reduces knee pain and bone marrow lesions over 1 year: a randomised controlled trial. Ann Rheum Dis 2012;71:1322-8

29. Herrero-Beaumont G, Roman-Blas JA, Largo R, et al. Bone mineral density and joint cartilage: four clinical settings of a complex relationship in osteoarthritis. Ann Rheum Dis 2011;70:

1523-5.

30. Pelletier J-P, Kapoor M, Fahmi H, et al. Strontium ranelate reduces the progression of experimental dog osteoarthritis by inhibiting the expression of key proteases in cartilage and of interleukin-1 $\beta$ in the synovium. Ann Rheum Dis 2013;72:250-7.

31. Intema F, Sniekers YH, Weinans $\mathrm{H}$, et al. Similarities and discrepancies in subchondral bone structure in two differently induced canine models of osteoarthritis. J Bone Miner Res 2010;25:1650-7.

32. Intema F, Hazewinkel HA, Gouwens $D$, et al. In early $O A$, thinning of the subchondral plate is directly related to cartilage damage: results from a canine ACLT-meniscectomy model. Osteoarthritis Cartilage 2010;18:691-8.

33. Li Y, Li J, Zhu S, et al. Effects of strontium on proliferation and differentiation of rat bone marrow mesenchymal stem cells. Biochem Biophys Res Commun 2012;418:725-30.

34. Boileau C, Martel-Pelletier J, Abram F, et al. Magnetic resonance imaging can accurately assess the long-term progression of knee structural changes in experimental dog osteoarthritis. Ann Rheum Dis 2008;67:926-32.
35. Blake GM, Compston JE, Fogelman I. Could strontium ranelate have a synergistic role in the treatment of osteoporosis? Commentary. J Bone Miner Res 2009;24:1354-57.

36. Riddle DL, Moxley G, Dumenci L. Associations between statin use and changes in pain, function and structural progression: a longitudinal study of persons with knee osteoarthritis. Ann Rheum Dis 2013;72:196-203.

37. Clockaerts S, Van Osch GJ, Bastiaansen-Jenniskens YM, et al. Statin use is associated with reduced incidence and progression of knee osteoarthritis in the Rotterdam study. Ann Rheum Dis

2012;11:642-7; Erratum in on page 1264 .

38. Abou-Raya A, Abou-Raya S, Helmii M, et al. Effect of low dose oral prednisolone on symptoms and systemic inflammation in older adults with moderate to severe knee osteoarthritis: a randomized placebo-controlled trial. New insights in osteoarthritis 2012; abstract OP0129, EULAR 2012. 